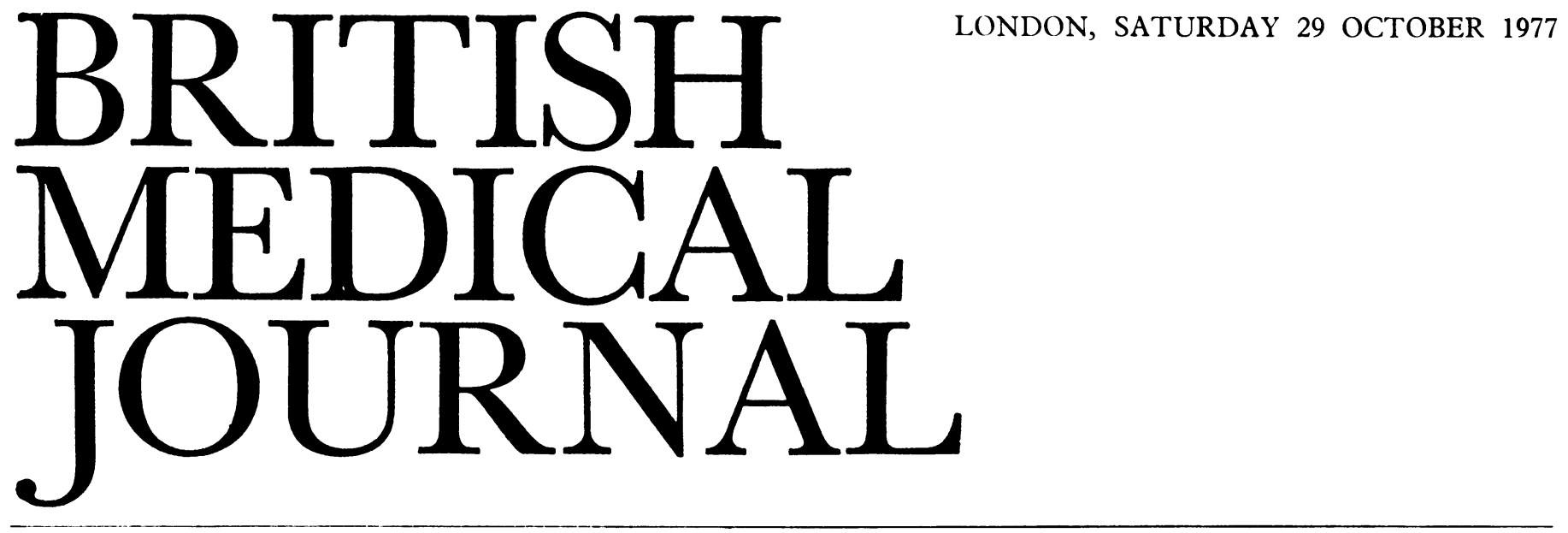

\title{
Management and diagnosis of children with the nephrotic syndrome
}

The prime feature of the nephrotic syndrome is gross proteinuria (selective to a varying degree) and resultant mirror-image hypoproteinaemia. Oedema is usual, as is hyperlipaemia; more variable characteristics include haematuria, systemic hypertension, nitrogen retention, and low plasma concentrations of the $\mathrm{C} 3$ fraction of complement.

Nephrotic syndrome may be congenital. The one relatively common example is the "Finnish" variety, a recessive autosomal genetic defect which presents at birth or soon afterwards with gross oedema. Affected children are extremely vulnerable to sudden death from overwhelming infection. Hallman's team from Helsinki discovered that by the 14th to 20 th week of pregnancy there is excess alphafetoprotein in the liquor and maternal plasma. ${ }^{1}$ At-risk families may now be monitored with the aim of selective abortion of the affected fetus. Earlier the same Finnish centre had attempted treatment with dialysis and early renal transplant, but the results were bad and the lives of these newborn infants grim.

Nephrotic syndrome may be secondary to a wide range of insults varying from heavy metals through collagen disease and quartan malaria to such rarities as renal venous thrombosis, snake bite, and troxidone treatment. Prevention by methods such as the removal of mercury from teething powders and the elimination of malaria is more effective than treatment, which is rarely satisfactory and in malarial nephropathy is of little avail.

Most cases of nephrotic syndrome in children are of the idiopathic variety, which affects $2 \cdot 1$ children per 100000 aged 0-12 years. Since 1955 percutaneous biopsy has greatly improved our knowledge of the light and electron microscopical changes and the fluorescent histological appearances; and in consequence prognosis has become more reliable, but it is salutary to remember that little therapeutic benefit has resulted. An era of improvement in treatment and prognosis began with penicillin in the mid-1940s and accelerated with corticosteroids, effective diuretics, and cytotoxic agents in the 1950 s; but remarkably little effective treatment has evolved since then.

Today management of idiopathic nephrotic syndrome begins with diagnosis and assessment of suitability for steroid treatment without prior biopsy or after biopsy if indicated. The diagnosis is based on typical gross proteinuria and hypoproteinaemia in a child aged over 6 months with no primary illness. In such cases steroid treatment is indicated without a prior renal biopsy unless there are clear indications for the procedure. These include the presence of haematuria, nitrogen retention, or systemic hypertension, an age of 8 years or more, a persistent low plasma C3 concentration, or normal or only slightly raised lipids. Even on such indications biopsy wil still show most children to have "minimal changes" on light microscopy. A minority will have the changes of membranous, membranoproliferative, proliferative, or chronic glomerulonephritis and require special consideration and expert advice on treatment (or avoidance of high-dosage continuous steroid treatment if membranoproliferative changes are present). The variant of minimal change histology in which there is focal segmental glomerulosclerosis carries a relatively bad prognosis.

All children in whom biopsy was not indicated and all with minimal change histology should receive a tapered continuous course of prednisolone orally. A suitable course for a 5-year-old is $60 \mathrm{mg}$ per day, $40 \mathrm{mg}$ per day, $20 \mathrm{mg}$ per day, and $10 \mathrm{mg}$ per day, reduction in dosage being at 10 -day intervals. ${ }^{2}$ Contact with chickenpox or measles must be avoided and immune globulins given if the patient becomes a contact. Potential incubation of a viral illness is a contraindication to starting steroid treatment. During treatment the child should be given a low sodium ( $<50 \mathrm{mmol}$ day), high protein $(3 \mathrm{~g} / \mathrm{kg}$ body weight day) diet, with calorie and carbohydrate intake restricted to normal levels for age. This helps to avoid excessive sodium retention, hypoproteinaemia, and cushingoid steroid obesity. A spectacular diuresis and loss of proteinuria occur in more than $90^{\circ} \%$ of such children within four weeks of starting treatment.

After 40 days treatment is stopped and results awaited. Of $>90 \%$ responders, roughly one-third will never relapse, one-third will relapse only infrequently (less than twice yearly), and one-third will relapse frequently (more than twice yearly and usually twice six-monthly). Non-relapsing responders need no further consideration, and infrequently relapsing responders need only a repeat of tapered continuous steroid treatment. Treatment should not be started for at least 14 days after the return of proteinuria unless oedema develops. Temporary self-resolving proteinuria may complicate respiratory infection in children freed of proteinuria and often resolves with no treatment.

Two important groups remain: frequent relapsers and the non-responders to steroid treatment. The treatment of 
frequent relapsers (two relapses in six months or four in one year) is not easy. Cyclophosphamide has proved very efficacious, ${ }^{3}$ in contrast to azathioprine, which has proved a disappointment. ${ }^{4}$ Unfortunately cyclophosphamide has many toxic effects, including haemorrhagic cystitis, marrow depression, reversible alopecia, chromosomal disturbances, and potential sterility. The care required to minimise these risks means that treatment is best given under expert supervision, and then only when further steroid treatment is inadvisable because of unacceptable osteoporosis, stunting of growth, obesity, or striae-effects which can be minimised by not giving prolonged continuous steroid treatment and by precautions on controlling diet. Most children do well even with two or three courses of steroid each year for a year or two; at some time a prolonged six months' course of alternateday treatment (20-30 mg prednisolone every second morning) may be needed. This seems to be a better regimen than three days on and four days off treatment and is certainly less toxic and more likely to permit growth than continuous treatment.

Even when steroid toxicity makes cyclophosphamide treatment necessary it should not begin until a diuresis has been produced by steroids. A high urine volume day and night must be continued throughout treatment and low dosage steroid (10-15 mg prednisolone a day) needs to be given to protect the bone marrow. Parents will accept reversible alopecia. Wigs help and so do photographs of previously bald patients with healthy hair again. When cyclophosphamide has been given (6-8 weeks at $2 \cdot 5-3 \mathrm{mg} \mathrm{kg}$ day) to steroid-responsive but steroid-toxic patients (almost universally with minimal change lesions) some $60^{\circ}{ }_{0}$ remain free of relapse after five years. ${ }^{3}$ This long period free of proteinuria enables the dwarfed, cushiongoid, osteoporotic patient to enjoy a long overdue growth spurt to catch up height and return to a normal, lean shape.

Non-responders to an initial 40-day course of steroid treatment should be assessed by percutaneous renal biopsy, if this has not already been done. Setting aside complex cases, most will show minimal changes with or without focal segmental glomerulosclerosis (FSGS). Those with no FSGS respond completely and spontaneously within a year. Those with FSGS usually do badly. ${ }^{6}$ A reasonable policy after failure of continuous high dosage steroid treatment to clear gross proteinuria is to delay retreatment for three months, maintaining freedom from oedema with diuretics such as frusemide (20-80 mg daily) and spironolactone $100 \mathrm{mg}$ daily. Infections of the urinary tract and elsewhere may be related to non-response, and peritonitis is an especial hazard. Early vigorous broad-spectrum antimicrobial cover (against Gramnegative organisms and streptococci) should be given at the first suspicion of infection. Delay is dangerous. Gramnegative septicaemia, shock, and death occur with frightening speed in children previously treated with steroids. Cyclophosphamide will accelerate the loss of proteinuria in children with minimal change lesions non-responsive to steroids, but its toxicity precludes its use in those circumstances.

Focal segmental glomerulosclerosis is commoner in older ( $>7$ years) girls and carries a poor prognosis. Steroid treatment may ameliorate proteinuria but rarely clears it, and symptomatic diuretic, dietetic, and antimicrobial treatment will be needed. Death may occur within a few years, as the initially scanty lesion spreads to affect a majority of glomeruli and destroy them. ${ }^{6}$ There is no evidence that cyclophosphamide (or any other drug) helps, and such children eventually require long-term haemodialysis or renal transplantation.

\footnotetext{
${ }^{1}$ Seppala, M, et al, Lancet, 1976, 2, 123.
}

2 Arneil, G C, Lancet, 1956, 1, 409.

${ }^{3}$ McDonald, J, Murphy, A V, and Arneil, G C, Lancet, 1974, 2, 980.

- Abramowicz, M, et al, Lancet, 1970, 1, 959.

${ }^{5}$ Barratt, T M, et al, Archives of Disease in Childhood, 1977, 52, 462.

${ }^{6}$ Churg, I, Habib, R, and White, R H R, Lancet, 1970, 1, 1299.

\section{Divers' ear}

Incapacitating ear pain caused by infection of the external canal is one of many important risks for North Sea divers. Traditionally acute otitis externa is associated with swimming - a view supported by Hoadley and $\mathrm{Knight}^{1}$ in their study of 244 people: $7 \cdot 3 \%$ of swimmers had ear complaints but only $3.1 \%$ of non-swimmers. Among 50 divers, however, Sperati and Pefumo $^{2}$ found that nearly half had ear problems compared with only three in a control group. Wright and Alexander ${ }^{3}$ showed that prolonged exposure to water changed the healthy ear flora from Gram-positive cocci and diphtheroids to Gram-negative bacilli and that this change often preceded acute symptoms. Alcock ${ }^{4}$ has now described how in 1974-5 two saturation dives in the North Sea had to be stopped and several others were disrupted by Pseudomonas aeruginosa ear infection affecting the diving team. Saturation dives, in which the divers stay at high atmospheric pressures, are planned to last anything from seven to 30 days or more. Severe ear pain caused by infection may make the demanding work impossible. With decompression and treatment the acute symptoms rapidly subside, but decompression is costly in both time and money and interferes with essential diving schedules.

In a typical saturation dive some six to eight men live inside a complex of several linked steel pressure chambers on the deck of a ship or oil rig. There is usually a main living chamber and a wash basin, shower, and lavatory as well as sleeping space. Divers move to and from the sea bed in a diving bell which can be keyed on to the pressure chamber through an air lock. The atmosphere is a pressurised mixture of helium and oxygen. The ambient temperature varies from 30 to $35^{\circ} \mathrm{C}$ and the relative humidity is $55 \%$ or more. Men descend in the bell to the sea bed, often for four to eight hours each day, typically for nine of 14 days in saturation. Decompression lasts for 50 hours or more. The complexity of the system, the arduous hours of work, and the waiting in cramped and humid conditions all increase the likelihood that opportunist pathogens will flourish.

To minimise the incidence of ear infection Alcock screened all divers before entering saturation, and those carrying Gramnegative organisms in their ears were excluded. Of 58 divers so screened, only one $(2 \%)$ carried $P$ aeruginosa. Seven days after entering the diving complex up to $70 \%$ harboured different species of Gram-negative organisms; $P$ aeruginosa was isolated at some stage from $82 \%$ of them. Some 10 of the 58 divers who were in saturation for up to 30 days developed pain in the ear and in eight of these $10 P$ aeruginosa was isolated. Ear swabs were collected by the divers themselves at two-day intervals during saturation, and as soon as these showed Gram-negative bacilli the men were treated with ear drops containing gentamicin and polymyxin $\mathrm{B}$; in the event, only two of the treated divers suffered pain. In addition to these measures an attempt was made to reduce contamination by Gram-negative organisms in the saturation chambers. This was found to be greatest in the washing and lavatory areas and the men's bedding. Intensive scrubbing of the chamber with detergent solution and the use of dichlorophen disinfectant 